Bull. Austral. Math. Soc.

$49 \mathrm{~J} 40,47 \mathrm{H} 19$

VOL. $62(2000) \quad[417-426]$

\title{
A PERTURBED ALGORITHM FOR STRONGLY NONLINEAR VARIATIONAL-LIKE INCLUSIONS
}

\author{
C.-H. Lee, Q.H. Ansari and J.-C. Yao
}

In this paper, we define the concept of $\eta$ - subdifferential in a more general setting than the one used by Yang and Craven in 1991. By using $\eta$-subdifferentiability, we suggest a perturbed algorithm for finding the approximate solutions of strongly nonlinear variational-like inclusions and prove that these approximate solutions converge to the exact solution. Several special cases are also discussed.

\section{INTRODUCTION}

Because of the applications in science, engineering and social sciences, the theory of variational inequalities has been extended and generalised in many different directions. An important and useful generalisation is variational-like inequality in the study of economic equilibrium problems. It has been studied by Ansari and Yao [2], Dien [3], Noor $[5,6]$, Parida et al [8], Siddiqi et al [11] and Yang and Chen [12] with further applications. Noor $[5,6]$ developed an auxiliary principle technique to prove the existence of solution for strongly nonlinear variational-like inequality and suggested an iterative algorithm for finding the approximate solution of this problem. Hassouni and Moudafi [4] used the proximal mapping technique to study a perturbed method for solving a new class of variational inclusions. Recently, Adly [1] considered a more general class of variational inclusions and used resolvent operator technique to prove a convergence result under some minimal hypotheses which are less demanding than the one used in [4]. In general we can not use resolvent operator or proximal mapping technique for studying a perturbed algorithm for finding the approximate solutions of variational-like inequalities. In this paper, we define the concept of $\eta$-subdifferential in a more general setting than the one used by Yang and Craven [13] which is also suitable to use to the so called proximal mapping technique to suggest a perturbed algorithm for finding the approximate solutions of a more general variational-like inequality, called a strongly nonlinear variational-like inclusion. We also prove that these approximate solutions converge to the exact solution. Several special cases are also discussed.

Received 23rd February, 2000

This research was supported by the National Science Council of the Republic of China.

Copyright Clearance Centre, Inc. Serial-fee code: 0004-9727/00 \$A2.00+0.00. 
Let $H$ be a real Hilbert space endowed with a norm $\|\cdot\|$ and inner product $\langle.,$.$\rangle .$ Given nonlinear operators $T, A: H \rightarrow H$ and $\eta: H \times H \rightarrow H$, we consider the following problem:

Problem 1. Find $x \in H$ such that $x \in \operatorname{dom} \phi$ and

$$
\langle T(x)-A(x), \eta(y, x)\rangle \geqslant \phi(x)-\phi(y), \quad \text { for all } y \in H,
$$

where $\phi: H \rightarrow \mathbb{R} \cup\{+\infty\}$ and $\operatorname{dom} \phi=\{z \in H: \phi(z)<\infty\}$. The inequality (1) is known as a strongly nonlinear variational-like inclusion.

SPECial Cases.

(I) If $\eta(y, x)=g(y)-g(x)$, where $g: H \rightarrow H$ is a nonlinear operator, then Problem 1 is equivalent to the following problem considered by Yao [15]: Find $x \in H$ such that

$$
\langle T(x)-A(x), g(y)-g(x)\rangle \geqslant \phi(x)-\phi(y), \quad \text { for all } y \in H .
$$

(II) If $\phi \equiv \delta_{K}$, the indicator function of the closed convex set $K$, then Problem 1 reduces to the problem of finding $x \in K$ such that

$$
\langle T(x)-A(x), \eta(y, x)\rangle \geqslant 0, \quad \text { for all } y \in K \text {. }
$$

This problem was introduced and studied by Noor [6].

(III) If $\phi \equiv \delta_{K}$ and $A \equiv 0$, then Problem 1 becomes to find $x \in K$ such that

$$
\langle T(x), \eta(y, x)\rangle \geqslant 0, \quad \text { for all } y \in K .
$$

This has been studied in $[5,8, \mathbf{1 1}, \mathbf{1 2}]$. The inequality (4) is known as a variational-like inequality.

(IV) If $\eta(y, x)=y-g(x)$, where $g: H \rightarrow H$ is a nonlinear operator, then Problem 1 is equivalent to a problem considered by Hassouni and Moudafi [4]: To find $x \in H$ such that $x \in \operatorname{dom} \phi$ and

$$
\langle T(x)-A(x), y-g(x)\rangle \geqslant \phi(x)-\phi(y), \quad \text { for all } y \in H .
$$

If $g(x)=x,(5)$ is similar to a problem over a closed convex set considered by Siddiqi, Ansari and Kazmi [10].

(V) If $\phi \equiv \delta_{K}$ and $\eta(y, x)=y-g(x)$, where $g: K \rightarrow K$ is a nonlinear operator, then the class of strongly nonlinear variational inequality problems given by

$$
\langle T(x)-A(x), y-g(x)\rangle \geqslant 0, \quad \text { for all } y \in K,
$$

is recovered from Problem 1. Such problems were studied by Siddiqi and Ansari [9] and Yao [14].

It is clear from these special cases that our Problem 1 is a more general unifying one, which is one of the main motivations for this paper. 


\section{2. $\eta$-SUBDIFFERENTIAL}

We define the concept of $\eta$-subdifferential in a more general setting than that given in [13]. This notion of $\eta$-subdifferential will be used in our perturbed iterative algorithm for finding the approximate solution of variational-like inclusion (1).

Let $\eta: H \times H \rightarrow H$ and $\phi: H \rightarrow \mathbb{R} \cup\{+\infty\}$. A vector $w \in H$ is called an $\eta$-subgradient of $\phi$ at $x \in \operatorname{dom} \phi$ if

$$
\langle w, \eta(y, x)\rangle \leqslant \phi(y)-\phi(x), \quad \text { for all } y \in H \text {. }
$$

We can associate with each $\phi$ the $\eta$-subdifferential map $\partial_{\eta} \phi$ defined by

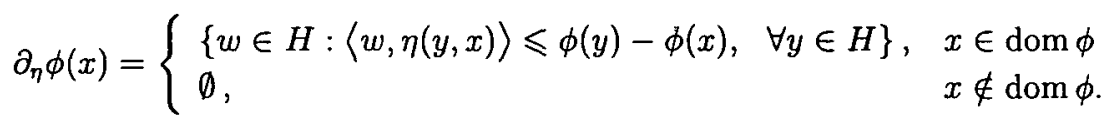

For $x \in \operatorname{dom} \phi, \partial_{\eta} \phi(x)$ is called the $\eta$-subdifferential of $\phi$ at $x$.

In the definition of $\eta$-subdifferential in the sense of Yang and Craven [13], the function $\phi$ needs to be local Lipschitz and can not take the value $+\infty$. The following example shows that our definition of $\eta$-subdifferential is more general than that in [13].

EXAMPLE. Let $\phi: \mathbb{R} \rightarrow \mathbb{R} \cup\{+\infty\}$ be defined by

$$
\phi(x)= \begin{cases}x, & x \leqslant 0 \\ +\infty, & x>0\end{cases}
$$

and $\eta: \mathbb{R} \times \mathbb{R} \rightarrow \mathbb{R}$ by

$$
\eta(x, y)=x+2 y \text {. }
$$

Then we have $\partial_{\eta} \phi(x)=[1, \infty), x \in \operatorname{dom} \phi$.

Let $x \in \operatorname{dom} \phi$, that is, $\phi(x)=x$ and $x \leqslant 0$. If $w \in \partial_{\eta} \phi(x)$, then

$$
w(y+2 x) \leqslant \phi(y)-x, \quad \text { for all } y \in \mathbb{R} .
$$

Since $\phi(y)=+\infty$, for $y>0$, we thus have

$$
w(y+2 x) \leqslant y-x, \text { for all } y \leqslant 0 .
$$

If $x=0$, then $w(y) \leqslant y$, for $y \leqslant 0$ and thus $w \geqslant 1$. If $x<0$, then

$$
w \geqslant \frac{y-x}{y+2 x}, \quad \text { for all } y \leqslant 0 \text {. }
$$

Letting $y \rightarrow-\infty$, we have $w \geqslant 1$. In any case, we have $w \geqslant 1$, that is, $\partial_{\eta} \phi(x) \subseteq$ $[1, \infty)$, for all $x \leqslant 0$. Now let $w \in[1, \infty)$. If $w \notin \partial_{\eta} \phi(x)$ for some $x_{0} \leqslant 0$, then there exists $y_{0} \leqslant 0$ such that

$$
w\left(y_{0}+2 x_{0}\right)>y_{0}-x_{0}
$$


It is clear that the case $x_{0}=y_{0}=0$ can not happen. If $x_{0}<0$ and $y_{0}=0$, then we have $w<-1 / 2$ which is a contradiction. If $x_{0}=0$ and $y_{0}<0$, then we have $w<1$ which is also a contradiction. Finally, if $x_{0}<0$ and $y_{0}<0$, then

$$
1 \leqslant w<\frac{y_{0}-x_{0}}{y_{0}+2 x_{0}}
$$

from which it follows that $x_{0}>0$ and this is also a contradiction. In our claim, we have $[1, \infty) \subseteq \partial_{\eta} \phi(x)$, for all $x \leqslant 0$. Consequently, $\partial_{\eta} \phi(x)=[1, \infty)$, for all $x \leqslant 0$.

TheOREM 1. Let $\phi: H \rightarrow \mathbb{R} \cup\{+\infty\}$ be nontrivial, that is, dom $\phi \neq \emptyset$. Then $x \in H$ is a solution of Problem 1 if and only if $x \in \operatorname{dom} \phi$ and $A(x)-T(x) \in \partial_{\eta} \phi(x)$.

PROOF: This directly follows from the definition of the $\eta$-subdifferential.

Let us recall the following definitions.

DEFinition 1: An operator $T: H \rightarrow H$ is called:

(i) strongly monotone, if there exists a constant $\alpha \geqslant 0$ such that

$$
\langle T(x)-T(y), x-y\rangle \geqslant \alpha\|x-y\|^{2}, \quad \text { for all } x, y \in H
$$

(ii) Lipschitz continuous, if there exists a constant $\beta>0$ such that

$$
\|T(x)-T(y)\| \leqslant \beta\|x-y\|, \quad \text { for all } x, y \in H .
$$

It is clear that $\alpha \leqslant \beta$.

DEFINITION 2: $[5,6]$ An operator $\eta: H \times H \rightarrow H$ is called:

(i) monotone, if

$$
\langle x-y, \eta(x, y)\rangle \geqslant 0, \quad \text { for all } x, y \in H
$$

(ii) strictly monotone, if the equality holds in (8) only when $x=y$.

(iii) strongly monotone, if there exists a constant $\sigma \geqslant 0$ such that

$$
\langle x-y, \eta(x, y)\rangle \geqslant \sigma\|x-y\|^{2}, \quad \text { for all } x, y \in H
$$

(iv) Lipschitz continuous, if there exists a constant $\delta>0$ such that

$$
\|\eta(x, y)\| \leqslant \delta\|x-y\|, \quad \text { for all } x, y \in H .
$$

When $\eta(x, y)=T(x)-T(y)$ for $T: H \rightarrow H$, then Definition 2 ((iii) and (iv)) reduce to Definition 1 ((i) and (ii), respectively). In particular, we have $\sigma \leqslant \delta$.

Assumption 1. The operator $\eta: H \times H \rightarrow H$ satisfies the condition

$$
\eta(y, x)+\eta(x, y)=0, \quad \text { for all } x, y \in H .
$$


Let $Q: H \rightarrow 2^{H}$ be a multivalued map. Then the graph of $Q$, denoted by $\operatorname{Graph}(Q)$, is defined as follows:

$$
\operatorname{Graph}(Q)=\{(x, y) \in H \times H: y \in Q(x)\} .
$$

Definition 3: Let $\eta: H \times H \rightarrow H$ be a given map. Then a multivalued map $Q: H \rightarrow 2^{H}$ is called $\eta$-monotone, if for all $x, y \in H$,

$$
\langle u-v, \eta(x, y)\rangle \geqslant 0, \quad \text { for all } u \in Q(x), v \in Q(y) .
$$

$Q$ is called maximal $\eta$-monotone if and only if it is $\eta$-monotone and there is no other $\eta$-monotone multivalued map whose graph strictly contains the $\operatorname{Graph}(Q)$.

The proof of the following lemma is on the lines of the proof of [16, Lemma 3] and therefore we omit it.

Proposition 1. Let $\eta: H \times H \rightarrow H$ be a map. A multivalued map $Q$ is maximal $\eta$-monotone if and only if $Q$ is $\eta$-monotone and it follows from $(x, u) \in H \times H$ and

$$
\langle u-v, \eta(x, y)\rangle \geqslant 0, \quad \text { for all }(y, v) \in \operatorname{Graph}(Q)
$$

that $(x, u) \in \operatorname{Graph}(Q)$.

Proposition 2. Let $\eta: H \times H \rightarrow H$ be strictly monotone and $Q: H \rightarrow 2^{H}$ be an $\eta$-monotone multivalued map. If the range of $(I+\lambda Q), R(I+\lambda Q)=H$, for $\lambda>0$ where $I$ is the identity operator, then $Q$ is maximal $\eta$-monotone. Furthermore, the inverse operator $(I+\lambda Q)^{-1}: H \rightarrow H$ is single-valued.

Proof: Suppose that $Q$ is not a maximal $\eta$-monotone, then there exists $\left(x_{0}, u_{0}\right) \notin$ $\operatorname{Graph}(Q)$ such that

$$
\left\langle u_{0}-v, \eta\left(x_{0}, y\right)\right\rangle \geqslant 0, \quad \text { for all }(y, v) \in \operatorname{Graph}(Q) .
$$

By assumption that $R(I+\lambda Q)=H$, there exists $\left(x_{1}, u_{1}\right) \in \operatorname{Graph}(Q)$ such that

$$
x_{1}+\lambda u_{1}=x_{0}+\lambda u_{0} .
$$

Since (9) is true for all $(y, v) \in \operatorname{Graph}(Q)$, we have

$$
\left\langle u_{0}-u_{1}, \eta\left(x_{0}, x_{1}\right)\right\rangle \geqslant 0 .
$$

But from (10), we have $\lambda\left(u_{0}-u_{1}\right)=x_{1}-x_{0}$ and hence

$$
\frac{1}{\lambda}\left\langle x_{1}-x_{0}, \eta\left(x_{0}, x_{1}\right)\right\rangle \geqslant 0 \text {. }
$$

Multiplying by $\lambda>0$, we have

$$
\left\langle x_{0}-x_{1}, \eta\left(x_{0}, x_{1}\right)\right\rangle \leqslant 0 .
$$


Since $\eta$ is strictly monotone, we have $x_{0}=x_{1}$ and hence from (10) we get $u_{1}=u_{0}$. So, we reach the contradiction that $\left(x_{1}, u_{1}\right) \in \operatorname{Graph}(Q)$ or $\left(x_{0}, u_{0}\right) \in \operatorname{Graph}(Q)$. Therefore $Q$ is maximal $\eta$-monotone.

For the second part, let $x, y \in(I+\lambda Q)^{-1}(z)$. Then $(z-x) / \lambda \in Q(x)$ and $(z-y) / \lambda \epsilon$ $Q(y)$. We set $u=(z-x) / \lambda$ and $v=(z-y) / \lambda$. Therefore, $z=\lambda u+x$ and $z=\lambda v+y$. By $\eta$-monotonicity of $Q$, we have

$$
\begin{aligned}
0=\langle z-z, \eta(x, y)\rangle & =\langle\lambda u+x-(\lambda v+y), \eta(x, y)\rangle \\
& =\lambda\langle u-v, \eta(x, y)\rangle+\langle x-y, \eta(x, y)\rangle \\
& \geqslant\langle x-y, \eta(x, y)\rangle .
\end{aligned}
$$

Since $\eta$ is strictly monotone, we have $x=y$. And hence $(I+\lambda Q)^{-1}$ is a single-valued map.

OPEN PROBLEM: If $Q$ is maximal $\eta$-monotone then under what conditions do we have $R(I+\lambda Q)=H ?$

The following lemma can be easily proved and therefore we omit its proof.

Lemma 1. Let $\eta: H \times H \rightarrow H$ satisfy Assumption 1 and $\phi: H \rightarrow \mathbb{R} \cup\{+\infty\}$. Then the multivalued map $\partial_{\eta} \phi: H \rightarrow 2^{H}$ is $\eta$-monotone.

\section{Perturbed iterative algorithm}

Throughout this section, we shall assume that $\eta: H \times H \rightarrow H$ is strictly monotone and satisfies Assumption 1 and $\phi: H \rightarrow \mathbb{R} \cup\{+\infty\}$ such that $R\left(I+\lambda \partial_{\eta} \phi\right)=H$ for $\lambda>0$.

From Proposition 1 and Lemma 1, we note that the mapping

$$
J_{\lambda}^{\phi}(x):=\left(I+\lambda \partial_{\eta} \phi\right)^{-1}(x), \quad \text { for all } x \in H
$$

is single-valued.

Let us transform (1) into a fixed point problem.

LEMMA $2 . x$ is a solution of (1) if and only if it satisfies the relation

$$
x=J_{\lambda}^{\phi}(x-\lambda(T(x)-A(x))),
$$

where $\lambda>0$ is a constant, $J_{\lambda}^{\phi}=\left(I+\lambda \partial_{\eta} \phi\right)^{-1}$ is the so-called proximal map and I stands for identity operator on $H$.

Proof: From the definition of $J_{\lambda}^{\phi}$, we have

$$
x-\lambda(T(x)-A(x)) \in x+\lambda \partial_{\eta} \phi(x)
$$

and hence

$$
A(x)-T(x) \in \partial_{\eta} \phi(x)
$$


By using the definition of the $\eta$-subdifferential, we have

$$
\langle A(x)-T(x), \eta(y, x)\rangle \leqslant \phi(y)-\phi(x), \quad \text { for all } y \in H .
$$

This implies that $x$ is a solution of (1).

Lemma 2 enables us to reformulate Problem 1 as the fixed point problem of solving

$$
x=F(x)
$$

where

$$
F(x)=J_{\lambda}^{\phi}(x-\lambda(T(x)-A(x))) .
$$

On the basis of this observation, we have the following algorithm to find an approximate solution of (1).

Algorithm. Given $x_{0} \in H$, compute $x_{n+1}$ by the rule

$$
x_{n+1}=J_{\lambda}^{\phi}\left(x_{n}-\lambda\left(T\left(x_{n}\right)-A\left(x_{n}\right)\right)\right),
$$

where $\lambda>0$ is a constant.

To perturb scheme (13), first we add in the right hand side of (13) an error $e_{n}$ to take into account a possible inexact computation of the proximal point and we consider another perturbation by replacing $\phi$ in (13) by $\phi_{n}$, where the sequence $\left\{\phi_{n}\right\}$ approximates $\phi$. Finally, we obtain the perturbed algorithm which generates from any starting point $x_{0} \in H$ a sequence $\left\{x_{n}\right\}$ by the rule

$$
x_{n+1}=J_{\lambda}^{\phi_{n}}\left(x_{n}-\lambda\left(T\left(x_{n}\right)-A\left(x_{n}\right)\right)\right)+e_{n} .
$$

Lemma 3. Let $\eta: H \times H \rightarrow H$ be strongly monotone and Lipschitz continuous with constants $\sigma>0$ and $\delta>0$, respectively, which satisfies Assumption 1. Then

$$
\left\|J_{\lambda}^{\phi}(x)-J_{\lambda}^{\phi}(y)\right\| \leqslant \tau\|x-y\|, \quad \text { for all } x, y \in H
$$

where $\tau=\delta / \sigma$.

Proof: From the definition of $J_{\lambda}^{\phi}$, we have

$$
J_{\lambda}^{\phi}(x)=\left(I+\lambda \partial_{\eta} \phi\right)^{-1}(x)
$$

and hence

$$
\frac{1}{\lambda}\left(x-J_{\lambda}^{\phi}(x)\right) \in \partial_{\eta} \phi\left(J_{\lambda}^{\phi}(x)\right)
$$

and

$$
\frac{1}{\lambda}\left(y-J_{\lambda}^{\phi}(y)\right) \in \partial_{\eta} \phi\left(J_{\lambda}^{\phi}(y)\right), \quad \text { for all } x, y \in H
$$

Since $\partial_{\eta} \phi$ is $\eta$-monotone, we have

$$
\frac{1}{\lambda}\left\langle x-J_{\lambda}^{\phi}(x)-\left(y-J_{\lambda}^{\phi}(y)\right), \eta\left(J_{\lambda}^{\phi}(x), J_{\lambda}^{\phi}(y)\right)\right\rangle \geqslant 0 .
$$


Multiplying by $\lambda>0$, we get

$$
\left\langle x-y-\left(J_{\lambda}^{\phi}(x)-J_{\lambda}^{\phi}(y)\right), \eta\left(J_{\lambda}^{\phi}(x), J_{\lambda}^{\phi}(y)\right)\right\rangle \geqslant 0,
$$

or

$$
\left\langle x-y, \eta\left(J_{\lambda}^{\phi}(x), J_{\lambda}^{\phi}(y)\right)\right\rangle \geqslant\left\langle\left(J_{\lambda}^{\phi}(x)-J_{\lambda}^{\phi}(y)\right), \eta\left(J_{\lambda}^{\phi}(x), J_{\lambda}^{\phi}(y)\right)\right\rangle .
$$

Since $\eta(.,$.$) is strongly monotone, we have$

$$
\left\langle\left(J_{\lambda}^{\phi}(x)-J_{\lambda}^{\phi}(y)\right), \eta\left(J_{\lambda}^{\phi}(x), J_{\lambda}^{\phi}(y)\right)\right\rangle \geqslant \sigma\left\|J_{\lambda}^{\phi}(x)-J_{\lambda}^{\phi}(y)\right\|^{2} .
$$

From Lipschitz continuity of $\eta(\cdot, \cdot)$, we get

$$
\begin{aligned}
\left\langle x-y, \eta\left(J_{\lambda}^{\phi}(x), J_{\lambda}^{\phi}(y)\right)\right\rangle & \leqslant\|x-y\|\left\|\eta\left(J_{\lambda}^{\phi}(x), J_{\lambda}^{\phi}(y)\right)\right\| \\
& \leqslant \delta\|x-y\|\left\|J_{\lambda}^{\phi}(x)-J_{\lambda}^{\phi}(y)\right\| .
\end{aligned}
$$

By combining inequalities (15), (16) and (17), we have

$$
\left\|J_{\lambda}^{\phi}(x)-J_{\lambda}^{\phi}(y)\right\| \leqslant \tau\|x-y\|, \quad \text { for all } x, y \in H,
$$

where $\tau=\delta / \sigma$.

Now we are ready to prove the main result of this paper.

THEOREM 2. Let $T: H \rightarrow H$ and $\eta: H \times H \rightarrow H$ be both Lipschitz continuous and strongly monotone and satisfy Assumption 1. Let $A: H \rightarrow H$ be Lipschitz continuous. For each $n$, let $\phi_{n}: H \rightarrow \mathbb{R} \cup\{+\infty\}$ and $\phi: H \rightarrow \mathbb{R} \cup\{+\infty\}$ be such that $R\left(I+\lambda \partial_{\eta} \phi_{n}\right)=R\left(I+\lambda \partial_{\eta} \phi\right)=H$ for $\lambda>0$. Assume that $\lim _{n \rightarrow+\infty}\left\|J_{\lambda}^{\phi_{n}}(z)-J_{\lambda}^{\phi}(z)\right\|=0$, for all $z \in H,\left\{x_{n}\right\}$ is generated by (14) with $\lim _{n \rightarrow+\infty}\left\|e_{n}\right\|=0$ and $x$ is a solution of (1). Then $x_{n+1}$ strongly converges to $x$, for

$$
\begin{aligned}
& \left|\lambda-\frac{(\alpha-\tau \rho)}{\beta^{2}-\rho^{2}}\right|<\frac{\sqrt{(\tau \rho-\alpha)^{2}-\left(\beta^{2}-\rho^{2}\right)\left(1-\tau^{2}\right)}}{\beta^{2}-\rho^{2}}, \\
& \alpha<\tau \rho-\sqrt{\left(\beta^{2}-\rho^{2}\right)\left(1-\tau^{2}\right)}, \quad \beta>\rho \text { and } \tau<1,
\end{aligned}
$$

where $\beta, \rho$ and $\delta$ are Lipschitz constants of $T, A$ and $\eta$, respectively, and $\alpha$ and $\sigma>0$ are strongly monotonicity constants of $T$ and $\eta$, respectively, with $\tau=\delta / \sigma$.

Proof: From Lemma 2, we see that $x \in H$ satisfying (1) is a solution of (11) and conversely. Thus, we have

$$
x=J_{\lambda}^{\phi}(x-\lambda(T(x)-A(x))) .
$$

By setting $h(x):=x-\lambda(T(x)-A(x))$ and from (11) and (14), we have

$$
\begin{aligned}
\left\|x_{n+1}-x\right\| & \leqslant\left\|J_{\lambda}^{\phi_{n}}\left(x_{n}-\lambda\left(T\left(x_{n}\right)-A\left(x_{n}\right)\right)\right)-J_{\lambda}^{\phi}(x-\lambda(T(x)-A(x)))\right\|+\left\|e_{n}\right\| \\
& =\left\|J_{\lambda}^{\phi_{n}}\left(h\left(x_{n}\right)\right)-J_{\lambda}^{\phi}(h(x))\right\|+\left\|e_{n}\right\| .
\end{aligned}
$$


By introducing the term $J_{\lambda}^{\phi_{n}}(h(x))$, we get

$$
\left\|J_{\lambda}^{\phi_{n}}\left(h\left(x_{n}\right)\right)-J_{\lambda}^{\phi}(h(x))\right\| \leqslant\left\|J_{\lambda}^{\phi_{n}}\left(h\left(x_{n}\right)\right)-J_{\lambda}^{\phi_{n}}(h(x))\right\|+\left\|J_{\lambda}^{\phi_{n}}(h(x))-J_{\lambda}^{\phi}(h(x))\right\| .
$$

By Lemma 3, we have

$$
\left\|J_{\lambda}^{\phi_{n}}\left(h\left(x_{n}\right)\right)-J_{\lambda}^{\phi}(h(x))\right\| \leqslant \tau\left\|h\left(x_{n}\right)-h(x)\right\|+\left\|J_{\lambda}^{\phi_{n}}(h(x))-J_{\lambda}^{\phi}(h(x))\right\| .
$$

Hence

$$
\begin{gathered}
\left.\left\|J_{\lambda}^{\phi_{n}}\left(h\left(x_{n}\right)\right)-J_{\lambda}^{\phi}(h(x))\right\| \leqslant \tau \| x_{n}-\lambda\left(T\left(x_{n}\right)\right)-A\left(x_{n}\right)\right)-x+\lambda(T(x)-A(x)) \| \\
+\left\|J_{\lambda}^{\phi_{n}}(h(x))-J_{\lambda}^{\phi}(h(x))\right\| \\
\leqslant \tau\left\|x_{n}-x-\lambda\left(T\left(x_{n}\right)-T(x)\right)\right\|+\tau \lambda\left\|A\left(x_{n}\right)-A(x)\right\| \\
+\quad+\left\|J_{\lambda}^{\phi_{n}}(h(x))-J_{\lambda}^{\phi}(h(x))\right\| .
\end{gathered}
$$

By using Lipschitz continuity and strongly monotonicity of $T$ (see [6]), we have

$$
\left\|x_{n}-x-\lambda\left(T\left(x_{n}\right)-T(x)\right)\right\|^{2} \leqslant\left(1+\lambda^{2} \beta^{2}-2 \lambda \alpha\right)\left\|x_{n}-x\right\|^{2} .
$$

By using Lipschitz continuity of $A$, we get

$$
\begin{gathered}
\left\|J_{\lambda}^{\phi_{n}}\left(h\left(x_{n}\right)\right)-J_{\lambda}^{\phi}(h(x))\right\| \leqslant\left(\tau \sqrt{\left(1+\lambda^{2} \beta^{2}-2 \lambda \alpha\right)}\right)\left\|x_{n}-x\right\|+\tau \lambda \rho\left\|x_{n}-x\right\| \\
+\left\|J_{\lambda}^{\phi_{n}}(h(x))-J_{\lambda}^{\phi}(h(x))\right\| \\
\leqslant\left(\tau \sqrt{\left(1+\lambda^{2} \beta^{2}-2 \lambda \alpha\right)}+\tau \lambda \rho\right)\left\|x_{n}-x\right\| \\
+\left\|J_{\lambda}^{\phi_{n}}(h(x))-J_{\lambda}^{\phi}(h(x))\right\|
\end{gathered}
$$

From the above second inequality and by combining (18) and (19), we get

$$
\left\|x_{n+1}-x\right\| \leqslant \theta\left\|x_{n}-x\right\|+\left\|J_{\lambda}^{\phi_{n}}(h(x))-J_{\lambda}^{\phi}(h(x))\right\|+\left\|e_{n}\right\|,
$$

where $\theta=\tau \sqrt{\left(1+\lambda^{2} \beta^{2}-2 \lambda \alpha\right)}+\tau \lambda \rho<1$, for

$$
\begin{gathered}
\left|\lambda-\frac{(\alpha-\tau \rho)}{\beta^{2}-\rho^{2}}\right|<\frac{\sqrt{(\tau \rho-\alpha)^{2}-\left(\beta^{2}-\rho^{2}\right)\left(1-\tau^{2}\right)}}{\beta^{2}-\rho^{2}}, \\
\alpha<\tau \rho-\sqrt{\left(\beta^{2}-\rho^{2}\right)\left(1-\tau^{2}\right)}, \beta>\rho \text { and } \tau<1 .
\end{gathered}
$$

By setting $\varepsilon_{n}=\left\|J_{\lambda}^{\phi_{n}}(h(x))-J_{\lambda}^{\phi}(h(x))\right\|+\left\|e_{n}\right\|$, we can write $\left\|x_{n+1}-x\right\| \leqslant \theta\left\|x_{n}-x\right\|+\varepsilon_{n}$. Hence

$$
\left\|x_{n+1}-x\right\| \leqslant \theta^{n+1}\left\|x_{0}-x\right\|+\sum_{j=0}^{n} \theta^{j} \varepsilon_{n-j} .
$$

Since $\lim _{n \rightarrow+\infty} \varepsilon_{n}=0$, from Ortega and Rheinboldt [7], we have that $x_{n}$ converges strongly to $x$. 


\section{REFERENCES}

[1] S. Adly, 'Perturbed algorithms and sensitivity analysis for a general class of variational inclusions', J. Math. Anal. Appl. 201 (1996), 609-630.

[2] Q.H. Ansari and J.C. Yao, 'Pre-variational inequalities in Banach spaces', in Optimization: Techniques and Applications, (L. Caccetta, K.L. Teo, P.F. Siew, Y.H. Leung, L.S. Jennings and V. Rehbock, Editors) (Curtin University of Technology, Perth, W.A., 1998), pp. 1165-1172.

[3] N.D. Dien,, 'Some remarks on variational-like and quasivariational-like inequalities', Bull. Austral. Math. Soc. 46 (1992), 335-342.

[4] A. Hassouni and A. Moudafi, 'A perturbed algorithm for variational inclusions', J. Math. Anal. Appl. 185 (1994), 706-712.

[5] M.A. Noor, 'Variational-like inequalities', Optimization 30 (1994), 323-330.

[6] M.A. Noor, 'Nonconvex functions and variational inequalities', J. Optimiz. Theory Appl. 87 (1995), 615-630.

[7] J.M. Ortega and Rheinboldt, Iterative solution of nonlinear equations in several variables (Academic Press, New York, 1970).

[8] J. Parida, M. Sahoo and A. Kumar, 'A Variational-like inequality problem', Bull. Austral. Math. Soc. 39 (1989), 225-231.

[9] A.H. Siddiqi and Q.H. Ansari, 'General strongly nonlinear variational inequalities', $J$. Math. Anal. Appl. 166 (1992), 386-392.

[10] A.H. Siddiqi, Q.H. Ansari and K.R. Kazmi, 'On nonlinear variational inequalities', Indian J. Pure Appl. Math. 25 (1994), 969-973.

[11] A.H. Siddiqi, A. Khaliq and Q.H. Ansari, 'On variational-like inequalities', Ann. Sci. Math. Quebec 18 (1994), 39-48.

[12] X.Q. Yang and G.Y. Chen, 'A class of nonconvex functions and pre-variational inequalities', J. Math. Anal. Appl. 169 (1992), 359-373.

[13] X.Q. Yang and B.D. Craven, 'Necessary optimality conditions with a modified subdifferential', Optimization 22 (1991), 387-400.

[14] J.C. Yao, 'On the general variational inequality', J. Math. Anal. Appl. 174 (1993), 550-555.

[15] J.C. Yao, 'Existence of generalized variational inequalities', Oper. Res. Lett. 15 (1994), $35-40$.

[16] C.J. Zhang, Y.J. Cho and S.M. Wei, 'Variational inequalities and surjectivity for set-valued monotone mappings', Top. Meth. Nonlinear Anal. 12 (1998), 169-178.

Department of Applied Mathematics

National Sun Yat-sen University

Kaohsiung 804, Taiwan

Republic of China
Department of Mathematics Aligarh Muslim University Aligarh 202002 India

Department of Applied Mathematics

National Sun Yat-sen University

Kaohsiung 804, Taiwan

Republic of China 